\title{
Management of bladder exstrophy in an adolescent girl: a case report
}

\author{
Ni Made Putri Suastari, ${ }^{1}$ Ni Nyoman Margiani, ${ }^{1}$ Kadek Budi Santosa, ${ }^{2}$ Dewa Gde Mahiswara, ${ }^{1}$ Firman Parulian Sitanggang, ${ }^{1}$ \\ I Wayan Yudiana²
}

Check for updates

pISSN: 0853-1773 • elSSN: 2252-8083 https://doi.org/10.13181/mji.cr.215249 Med J Indones. 2022;31:70-4

Received: January 02, 2021

Accepted: May 22, 2021

Published online: August 03, 2021

Authors' affiliations:

'Department of Radiology, Faculty of

Medicine, Universitas Udayana, Sanglah

General Hospital, Bali, Indonesia,

${ }^{2}$ Department of Urology Surgery, Faculty of Medicine, Universitas Udayana,

Sanglah General Hospital, Bali, Indonesia

\section{Corresponding author:}

Kadek Budi Santosa

Department of Urology Surgery, Faculty

of Medicine, Universitas Udayana,

Sanglah General Hospital, Jalan

Diponegoro, Denpasar, 80113, Bali,

Indonesia

Tel/Fax: +62-361-229711 ext. 384/

+62-361-224206

E-mail: busanbsa@gmail.com

\begin{abstract}
Bladder exstrophy (BE) is a very rare congenital abnormality, especially in girls. We reported a 17-year-old girl with protruded bladder and urinary incontinence. Her vaginal orifice appeared stenotic and shifted anteriorly. She had an incomplete labia minora, short perineum, and anterior displacement of the anus. The pelvic radiography showed a pubic diastasis with a manta ray sign, and the ultrasonography examination showed a bicornuate uterus. She had undergone a functional reconstruction surgery with cystectomy, ileal conduit, appendectomy, and vaginoplasty along with the advancement of abdominal flap reconstruction with multiple Z-plasty. Functionally, she was able to urinate through the ileal conduit comfortably, had a regular menstrual cycle with minimal pain, had a wider vaginal canal, and had a smooth blood flow. Aesthetically, she had a good lower abdomen appearance, remained dry, and had an odorless urine. In clinical practice, this rare case report can provide additional knowledge and management of the same cases with effective results.
\end{abstract}

KEYWORDS adolescent, bicornuate uterine, bladder exstrophy, case management
Bladder exstrophy $(\mathrm{BE})$ is a very rare congenital abnormality of urogenital system. Globally, the incidence of BE is only around 1:30,000-50,000 live births with a ratio of 3:1 among males and females. ${ }^{1}$ A multicenter research analysis from the EuroScan in 2010 stated that out of 12 countries in Europe, there were only 19 cases of BE live births. ${ }^{2}$

Delayed presentation of BE at adolescent age is extremely rare as surgical management may cause problems due to renal function deterioration, difficulty in securing abdominal closure, and malignant potential of bladder remnant. However, some patients may not receive treatment during infancy due to lack of awareness, limited health care access, and poor socioeconomic conditions in developing countries.
The management of $B E$ is challenging, which requires a complex reconstructive surgery to achieve improvements in sexual and social functions. There are many controversies regarding bladder preservation, urinary diversion techniques, vaginoplasty, or osteotomy. ${ }^{3,4}$ Thus, we reported a case of BE in an adolescent girl who had undergone radical cystectomy with ileal conduit and vaginoplasty without osteotomy.

\section{CASE REPORT}

A 17-year-old girl came to the hospital (Bali province) with a mass in her lower abdomen that occurred since birth, along with urinary incontinence through a small hole located around the mass. 
Menarche began at 16 years old with a regular menstrual cycle but was accompanied by abdominal pain and a little blood discharge. Due to limited health care access as she lived in a small village in East Sumba, East Nusa Tenggara Province ( $\pm 833 \mathrm{~km}$ from Bali), she only went to a health facility assisted by a foundation and referred to the hospital last year. When she was 5 years old, she had sought treatment but did not perform any surgical intervention due to economic and geographical conditions. There was no previous history of other illnesses and no family history of the same disease.
Her physical examination showed a soft mass in the suprapubic region with a diameter of $8 \mathrm{~cm}$ and was not covered by the anterior abdominal wall. There was a $0.5 \mathrm{~cm}$ diameter hole in the ureter and a hole underneath the vagina with a bifid clitoris, incomplete labia minor, vaginal stenosis, and anterior displacement. The perineum was appeared shortened, and the anus was displaced anteriorly (Figure 1a). Despite the potential pelvic deformities, she had a normal gait.

Her renal function was within normal limits. Her pelvic radiography showed a $10.3 \mathrm{~cm}$ pubic
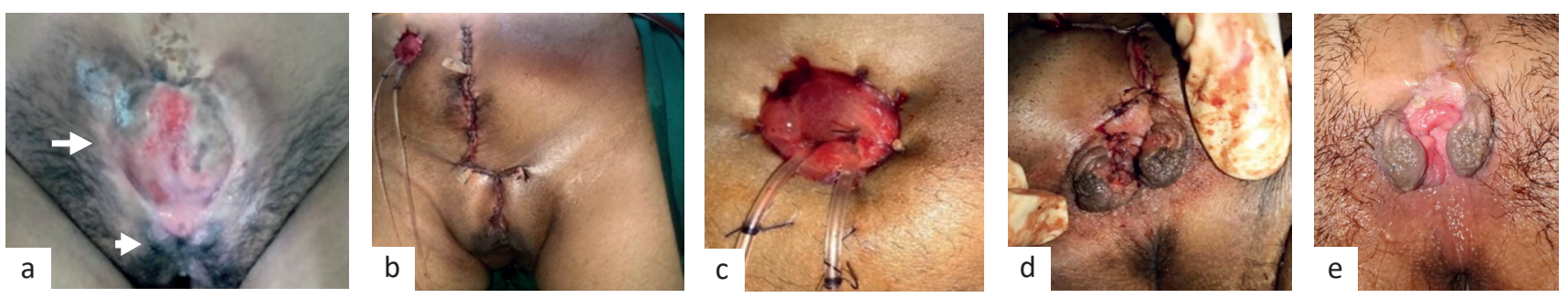

Figure 1. An adolescent girl with bladder exstrophy (BE). In preoperative, there was a mass in the suprapubic region, which was ureter (white arrow), and the hole underneath was a vagina (white arrowhead) with a bifid clitoris, incomplete labia minor, vaginal stenosis, and anterior displacement. The perineum was appeared shortened, and the anus was displaced anteriorly (a). In postoperative, the abdomen was closed (b), there was normal ileal conduit stoma (c), vaginal reconstruction (d), and vaginal orifice 1-month post-operatively (e)
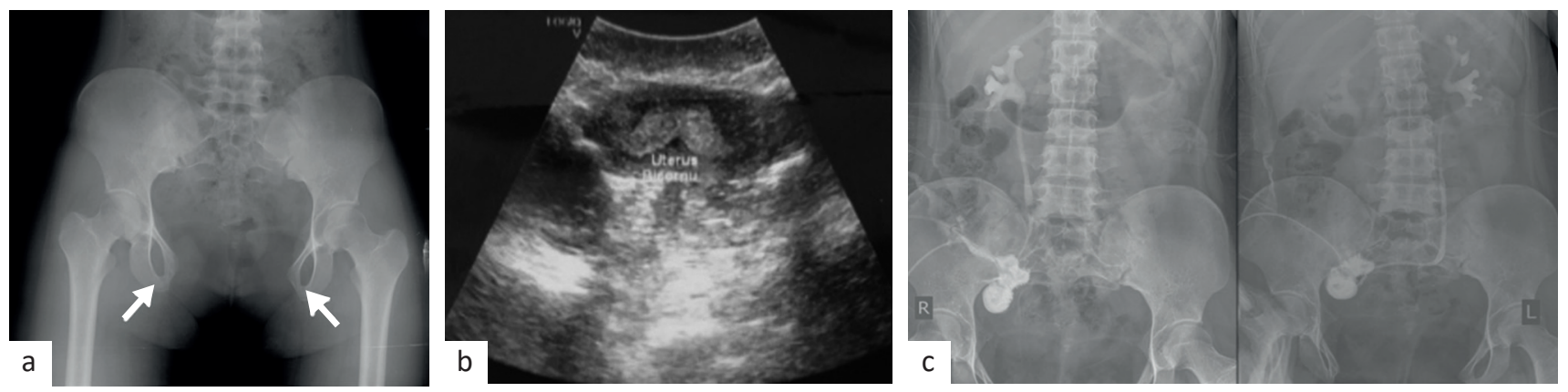

Figure 2. Radiological findings of pubic diastasis with a manta ray sign (a), bicornuate uterus (b), and splintography examination (c)
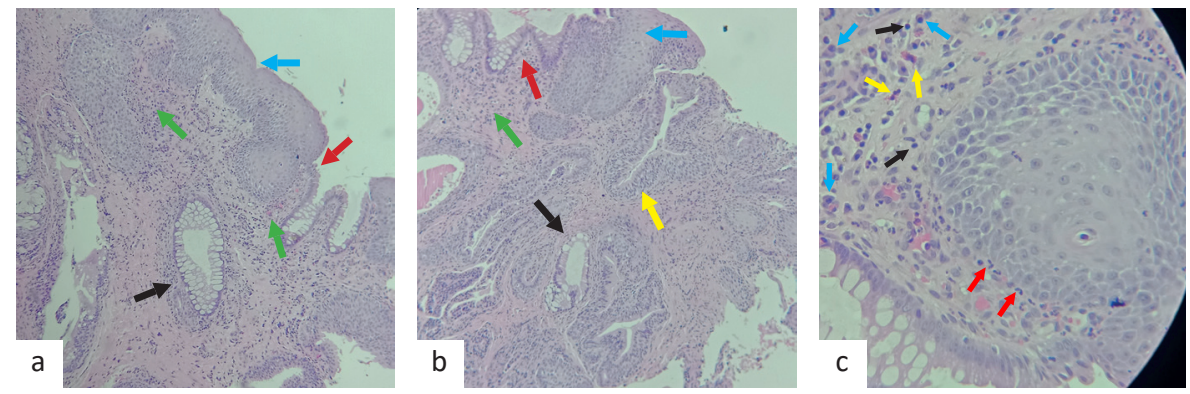

Figure 3. Histopathological examination of bladder mucosal tissue. Stratified squamous epithelium (blue arrow), stratified squamous epithelium with intestinal metaplasia (red arrow), intestinal metaplasia forming a glandular pattern (black arrow), inflammatory lymphoplasmacytic cells and neutrophils (green arrow), and transitional cells (yellow arrow) at 40× magnification ( $a$ and $b$ ). An enlargement of the previous image at 400x magnification emphasizing inflammatory cells with plasma cells (blue arrow), neutrophil cells (red arrow), eosinophil cells (yellow arrow), and lymphocyte cells (black arrow) (c) 
diastasis with a manta ray sign (Figure 2a), and her ultrasonography examination showed normal bilateral kidneys and retroflexed uterus sized about $6.6 \times 3 \mathrm{~cm}$, indicating bicornuate uterus (Figure $2 \mathrm{~b}$ ). The biopsy showed a connective tissue with squamous epithelium and transitional cells with glandular metaplasia and chronic suppurative inflammation (Figure 3).

\section{Surgical procedure}

She had undergone cystectomy, ileal conduit, appendectomy, and vaginoplasty along with the advancement of abdominal flap reconstruction with multiple Z-plasty in one setting. Cystectomy was performed with an elliptical incision of the abdominal wall around the exposed bladder area of $1 \mathrm{~cm}$ edge. The incision was continued to the intraperitoneal and extraperitoneal spaces. The ligation of the dorsal vein complex was done in the anteroinferior of the bladder and followed by the opening of the endopelvic fascia. The bladder was released by cutting both the distal ureters and ligation of the superior and inferior vesical vasa.

An appendectomy was performed along with the preparation of the intestinal segment for the ileal conduit (using 10-15 cm of terminal ileus segment, which is $20 \mathrm{~cm}$ from the ileocecal junction). Anastomosis of the ileum was done by end-to-end anastomosis with a stapler. Anastomosis on both ureters was then performed. The left ureter was crossed to the right side through the posterior peritoneum to the terminal ileal segment using Bricker procedure. Stoma was made on the right side of the abdominal wall. Vaginoplasty was performed by cutting the imperforated hymen and circumferential tissue on the posterior vaginal wall to facilitate hematocolpos evacuation. The anterior vaginal skin was separated, and the epithelium close to the symphysis from the underlying tissue and muscle and the tissue underneath were tightened to create the vaginal canal. Labiaplasty was performed by removing excess tissue, which further provides symmetrical labial skin. In the end, the abdominal surgical site was closed by rectus sheath flaps. The skin was closed by abdominal advancement flap reconstruction with multiple Z-plasty (Figure 1b).

After the procedure, her renal function test was normal. She was discharged 14 days after surgery. On day-24 after surgery, her splintography examination showed normal kidneys, ureters, and ileal conduits (Figure 2c). A month after surgery, she reported no complaint of continence through the ileal conduit, and the vaginal orifice looked wider than before but still needed further vaginal dilation. After 1 year of follow-up, her self-confidence, self-esteem, and social interactions had improved. She was able to maintain good social interaction with her family and community. She worked on the farm every day and was also active in socio-religious activities. She is well adapted because she can urinate through ileal conduit, which remained dry and had odorless urine. She had a regular menstrual cycle with minimal pain; her vaginal canal had widened, and the blood flowed smoothly. However, she admitted of being unmarried and never had sexual intercourse until this manuscript was prepared. The authors had obtained informed consent from the patient.

\section{DISCUSSION}

We reported a rare incidence of $B E$ in female, which mostly occurs in males. BE is a congenital abnormality in which the bladder opens on the lower abdominal wall since birth. BE can be accompanied by other disorders such as the urinary, genitalia, anorectal, and musculoskeletal systems. The pelvic radiography shows a manta ray sign which is the appearance of pubic symphysis diastasis. The ultrasound examination revealed retroflexion with a bicornuate uterus..$^{3-5}$ Massanyi et $\mathrm{al}^{6}$ reported that female with BE usually has a normal uterus and fallopian tubes, but there may be congenital abnormalities or uterine prolapse. Radiological examination plays an important role in detecting other anatomical abnormalities in BE.

The management of adolescent girls with $\mathrm{BE}$ is quite challenging; thus, a decision should be made whether to perform bladder preservation or cystectomy. Bladder preservation can be an option in the adolescent with $B E$ without significant histological changes or malignant transformation on the bladder mucosa and the possibility of adequate bladder capacity after bladder preservation to maintain voiding function and prevent vesicoureteral reflux (VUR). These patients require regular follow-ups to detect the early stage of malignant transformation and kidney function because a previous study has shown bladder preservation with VUR causes recurrent urinary tract infections that lead to kidney failure. ${ }^{7,8}$

In our case, we performed a cystectomy and an ileal conduit procedure due to small bladder capacity 
and low bladder compliance thus increasing intrabladder pressure which might lead to VUR. Small bladder capacity or slow growth is caused by growth factor alteration. This leads to the inability of urine reservation, increase of VUR risk, and secondary renal failure..$^{9,10}$ Shorter anatomy of distal ureter entering the bladder with a non-patent ureterovesical valve may also increase the risk of VUR. Moreover, an incompetent or short bladder neck accompanied by an inactive sphincter and affected bladder mucosa may produce inflammation or fibrosis or even leads to mucosal metaplasia and malignant transformation. ${ }^{9-11}$ Most patients with BE underwent cystectomy procedure because of failure to maintain bladder during the prior abdominal wall closure. ${ }^{10}$ Cystectomy procedure is an excellent choice for patients who live in a remote area due to follow-up restrain to evaluate renal function and symptomatic therapy associated with voiding dysfunction due to bladder preservation. Meanwhile, the ileal conduit technique for urinary diversion is easy to reconstruct and has managed to excrete urine of more than $90 \%$. Nerli et al ${ }^{11}$ reported that adult males with BE treated with cystectomy and ileal reservoir could urinate through ileal conduit, remained dry, and had an improved quality of life.

This patient had a small opening in the vaginal canal caused by the imperforated hymen. Vaginoplasty was performed to widen the vaginal introitus, which allows an insertion of two fingers. Cervellione et al $^{12}$ reported that a highly successful vaginoplasty in BE patients when performed in the second or third decade of life with low-risk complications for non-sexually active women. The reconstruction is considered safe and reliable to have a satisfactory outcome for cosmetic and sexual functions and prevent hematometrocolpos. ${ }^{12}$ Unfortunately, we could not assess the patient's sexual function because she was unmarried.

This patient had a normal gait, so the pubic bone reconstruction procedure was avoided since it may increase the risk of postoperative gait abnormality. In some cases, osteotomy may be an option considering the width of pubic diastasis and pelvic bone stiffness. Mansour et $\mathrm{al}^{13}$ reported that reconstruction without pelvic osteotomy also had good outcomes since it might result in orthopedic complications including nonunion of bone, pelvic asymmetry and stability, osteomyelitis, septic arthritis, and femoral nerve palsy.
Untreated bladder mucosa infection results in inflammation or fibrosis and can lead to mucosal metaplasia and malignancy. Hence, bladder mucosal biopsy was performed in this patient, which confirmed transitional cells with glandular metaplasia and chronic suppurative inflammation. BE had almost 700 times greater risk of bladder carcinoma, with a tendency to become adenocarcinoma (91\%) and epidermoid carcinoma (6\%). ${ }^{9}$

This patient had a chance to get pregnant as she had complete reproductive organs as evaluated by the obstetricians. Pregnancy in patient with $B E$ requires a multidisciplinary approach involving obstetricians and urological surgeons, as well as being managed in a tertiary care center. The most common recommendation for these patients is cesarean delivery due to the potential for continuing trauma during vaginal delivery or may result in previous urologic reconstructive injury or uterine prolapse. ${ }^{14}$

In conclusion, BE is a very rare congenital abnormality and infrequent in an adolescent girls. Radiologic evaluation plays an important role in the evaluation and detection of other anatomical abnormalities. Cystectomy in adolescent girls with $B E$ is an excellent choice for preserving kidney function and improving the social, sexual, and aesthetic functions, especially for patients in remote areas with limited health facilities.

\section{Conflict of Interest}

The authors affirm no conflict of interest in this study.

\section{Acknowledgment \\ None. \\ Funding Sources \\ None.}

\section{REFERENCES}

1. Fishel-Bartal M, Perlman S, Messing B, Bardin R, Kivilevitch $Z$, Achiron R, et al. Early diagnosis of bladder exstrophy: quantitative assessment of a low-inserted umbilical cord. J Ultrasound Med. 2017;36(9):1801-5.

2. Borer JG, Diamond DA. Bladder extrophy handbook. Boston: Boston Children's Hospital's. 2015. p. 1-32.

3. Kulkarni B, Chaudhari N. Embryogenesis of bladder extrophy: a new hypothesis. J Indian Assoc Pediatr Surg. 2008;13(2):57-60.

4. Ebert AK, Reutter $H$, Ludwig $M$, Rösch WH. The exstrophyepispadias complex. Orphanet J Rare Dis. 2009;4:23.

5. Lal NR, Borpatragohain DJ, Bhuyan MH. Bladder exstrophy: a rare case report. IOSR J Dent Med Sci. 2018;17(7):01-3.

6. Massanyi EZ, Gearhart JP, Kost-Byerly S. Perioperative management of classic bladder exstrophy. Res Rep Urol. 2013;5:67-75.

7. Apte AV, Saran R. Bladder exstrophy. People's J Sci Res. 
2009;2(2):35-9.

8. Baradaran N, Gearhart JP. Bladder exstrophy-epispadias-cloacal exstrophy complex: a contemporary overview. Neoreviews. 2010;11(12):e705-13.

9. Smeulders $\mathrm{N}$, Woodhouse CR. Neoplasia in adult exstrophy patients. BJU Int. 2001;87(7):623-8.

10. Ko JS, Lue K, Friedlander D, Baumgartner T, Stuhldreher $\mathrm{P}$, DiCarlo $\mathrm{HN}$, et al. Cystectomy in the pediatric exstrophy population: indications and outcomes. Urology. 2018;116:16871.

11. Nerli RB, Kamat GV, Alur SS, Koura A, Prabha V, Amarkhed SS.
Bladder exstrophy in adulthood. Indian J Urol. 2008;24(2):164-8.

12. Cervellione RM, Philips T, Baradaran N, Asanuma H, Mathews RI, Gearhart JP. Vaginoplasty in the female exstrophy population: outcomes and complications. J Pediatr Urol. 2010;6(6):595-9.

13. Mansour AM, Sarhan OM, Helmy TE, Awad B, Dawaba MS, Ghali AM. Management of bladder exstrophy epispadias complex in adults: is abdominal closure possible without osteotomy? World J Urol. 2010;28(2):199-204.

14. Amin KV, Tiwari N, Goel A, Chauhan AR. Pregnancy in case of exstrophy of bladder. Int J Reprod Contracept Obstet Gynecolo. 2017;6(4):1676-8. 